\title{
The potential diagnostic utility of coexpression of Ki-67 and P53 in the renal biopsy in pediatric lupus nephritis
}

This article was published in the following Dove Press journal: International Journal of Nephrology and Renovascular Disease

\author{
Mahtab H Rahbar' \\ Maryam R Rahbar ${ }^{2}$ \\ Nyousha Mardanpour ${ }^{1}$ \\ Sourena Mardanpour ${ }^{3}$ \\ 'Pathology Department, Iran \\ University of Medical Sciences, Tehran, \\ Iran; ${ }^{2}$ Nephrology department, Tehran \\ University of Medical Sciences, Tehran, \\ Iran; ${ }^{3}$ Santa Monica College, Santa \\ Monica, CA, USA
}

Background: The proliferative activity as well as apoptosis has been suggested to play a role in the pathogenesis of lupus nephritis (LN). The aim of the study was to investigate the coexpression of Ki-67-triggered marked proliferation and P53-induced apoptosis in renal biopsy of childhood lupus nephritis (cLN) and to compare the coexpression of proliferative and apoptotic indices between different subgroups and clinicopathologic patterns of renal disease.

Methods: Renal biopsy specimens of 33 children with lupus nephritis (LN) and 10 healthy subjects were retrospectively evaluated. The type of LN and activity and chronicity indices were determined. Ki-67 and P53 immunostaining were performed. The coexpression of Ki-67 and P53 was compared among different subgroups of LN and correlated with disease activity index, serum creatinine, proteinuria, anticardiolipin antibodies, and complement levels. Histopathological examination of LN was classified based on the International Society of Nephrology/ Renal Pathology. Histological LN activity was measured by the National Institutes of Health activity index (NIH-AI).

Results: In comparison with the healthy control group, the coexpression of Ki-67and P53 was greater in cLN (particularly in classes II, III, and IV) than in normal renal tissue. The coexpression of Ki-67and P53 shows a positive correlation with subclasses II, III, and IV of LN $(P<0.02)$ and LN activity index $(P<0.03)$. Moreover, the positive correlation was found between the coexpression of Ki-67 and P53 with erythrocyte sedimentation rate $(P<0.02)$, D-dimer $(P<0.03)$, serum creatinine $(P<0.03)$, proteinuria $(P<0.04)$, and anticardiolipin antibodies $(P<0.05)$ significantly. Unexpectedly, adverse correlation between the coexpression of Ki-67 and P53 with serum C3 $(P<0.02)$ and $\mathrm{C} 4$ complement $(P<0.03)$ was significant.

Conclusion: Our data showed that the coexpression of Ki-67-induced marked proliferation and P53-induced apoptosis in proliferative and active phases of cLN could reflect a valuable marker for treatment and remission in cLN patients before reaching the end stage of renal disease.

Keywords: Ki-67, P53, lupus nephritis, renal biopsy, children

\section{Introduction}

Childhood systemic lupus erythematosus (cSLE) is a severe multisystem autoimmune disease. Renal involvement occurs in the majority of cSLE patients and is a major cause of increased morbidity and mortality. Renal biopsy is an important investigative step in the management of childhood lupus nephritis (cLN). In $90 \%$ of patients, renal disease occurs within 2 years from the disease onset. ${ }^{1}$ Renal disease occurs in $50 \%-75 \%$ of all cSLE patients, mostly within the first 2 years after diagnosis. ${ }^{2}$ Lupus nephritis (LN) is more common in males and in non-white populations. ${ }^{3,4} \mathrm{LN}$ most
Correspondence: Mahtab Rahbar Pathology Department Iran University of Medical Sciences, Shahid Hemmat Highway, Tehran I4496I4535, Iran Tel+9891252I 40II

Email rahbarahbar@gmail.com 
commonly affects the glomerulus but can also involve the renal interstitium. Initial clinical manifestations of renal disease range from minimal proteinuria and microscopic hematuria to nephrotic-range proteinuria, urinary casts, severe hypertension, peripheral edema, and renal insufficiency or acute renal failure. On the contrary, the severity of nephritis may not correlate with the severity of the clinical signs and symptoms; a renal biopsy should be performed if there is any suspicion of glomerulonephritis, including persistent mild proteinuria. Routine performance of a renal biopsy has been advocated by some nephrologists in SLE patients with any signs of kidney disease. ${ }^{5,6}$ However, the renal biopsy is recognized as the gold standard model in deciding LN management in children. Renal biopsies should be reported as per the International Society of Nephrology/Renal Pathology classifications. Active and chronic glomerular lesions were reported based on the National Institutes of Health activity index (NIH-AI) score. ${ }^{7}$

Class I/II LN is milder and generally does not require immunosuppressive treatment, whereas class III/IV needs to be treated aggressively. ${ }^{8}$ Despite advances in treatment techniques, mortality related to SLE nephritis has been static over the last decade and morbidity continues to be a major factor. The outcome of $\mathrm{LN}$ is primarily dependent on histological classification at presentation. Hence, an early renal biopsy is strongly needed for early diagnosis of LN particularly in children with severe LN. Therefore, we will discuss some of the newer diagnostic markers that could be performed on renal biopsy specimens for on time diagnosis of proliferative and active phases of cLN, which is followed by medication. Control renal specimens were obtained from 10 healthy subjects matched for sex and age. The aim of the study was to show the relation of coexpression of Ki-67 and P53 on renal biopsy specimens with proliferative phase, disease activity, and clinicopathologic manifestations. The study protocol was approved by the local hospital ethics committee, Aliasghar hospital ethics and committee, and we confirmed that parents or legal guardians provided written informed consent for patients who were under the age of 18 years.

\section{Methods}

A retrospective study was conducted based on medical records from 33 pediatric patients with $\mathrm{LN}$ who had undergone percutaneous ultrasound-guided renal biopsy from 2010 to 2016 in a Pediatric Nephrology Tertiary Center of Ali Asghar Children Hospital at the Iran University of Medical Sciences. The patients ranged in age from 7 to 13 years (mean $8 \pm 1.3$ years). There were $21(63.5 \%)$ males and $12(36.5 \%)$ females, with a male to female ratio of 1.75:1. Renal biopsy should be considered in any patient with SLE who has a clinical or laboratory evidence of active nephritis, especially upon the first episode of nephritis and is not receiving any immunosuppressive or corticosteroid therapies. A few dubious cases that corresponded most likely to IgA/Henoch-Shönlein purpura nephropathy or membranoproliferative glomerulonephritis, with abundant deposition of immunoglobulin and complement factors, were excluded. No transplant biopsies were included. For those under steroid therapy, a washout period of 4 weeks for systemic treatment was allowed. Healthy renal specimens, as a control group, were obtained from subjects who had accidental traumatic nephrectomy. Specifically, demographic data (age, gender), clinical symptoms at presentation, laboratory findings such as erythrocyte sedimentation rate (ESR), antinuclear antibody (ANA), double-stranded DNA, anticardiolipin IgG, serum C3 and C4 levels, and histological diagnosis were obtained from all patients who underwent renal biopsy. All patients and/or their parents or legal guardians agreed to the experimental procedures and provided written informed consent. All procedures were approved by the ethics committee of Ali Asghar Children Hospital. All the data and personal information were made anonymous.

\section{Histopathological evaluation}

Biopsies were performed blindly under sterile conditions after ultrasonographic localization by using 16-gauge or 18-gauge biopsy needles. Two cores of tissue were taken each time under local anesthesia. The patients were kept flat in the supine position for 6 hours, and vital signs, hematuria, and hemoglobin levels were recorded. Patients were discharged at least 12 hours after the biopsy. Biopsies were fixed in $10 \%$ neutral formalin then paraffin-embedded blocks were prepared for each renal biopsy. Each renal biopsy was examined in randomly selected 10 high power fields using H\&E, Periodic Acid-Schiff, trichrome, and silver-stained specimens under high power (400×) magnification (field size of $0.274 \mathrm{~mm}^{2}$ and field diameter of $0.59 \mathrm{~mm}$ ) using a microscope with a wide angle. The presence of the following histopathological changes was analyzed according to the International Society of Nephrology/Renal Pathology Society (ISN/RPS) classification of LN (2003). ${ }^{2,4}$ Active and chronic glomerular lesions were reported based on the NIHAI score. ${ }^{7}$ Also, all kidney specimens were studied using IgG, IgA, IgM, C1q, C4, C3, and fibrinogen immunofluorescence 
antibody stain on paraffin-embedded tissue sections under high power $(400 \times)$ magnification (field size of $0.274 \mathrm{~mm}^{2}$ and field diameter of $0.59 \mathrm{~mm}$ ) by using immunofluorescence microscopy. All specimens were retrospectively evaluated by two neuropathologists.

\section{Immunohistochemical staining}

We analyzed the coexpression of $\mathrm{Ki}-67$ and P53 in renal biopsy of patients who were diagnosed for LN at Ali Asghar Children Hospital between 2010 and 2016. One $4 \mu \mathrm{m}$ section from each submitted paraffin block was first stained with $H \& E$ in order to verify that an adequate number of glomeruli were present and that the quality of fixation was sufficient for immunohistochemical analysis. Serial sections $(4 \mu \mathrm{m})$ were prepared from the selected blocks and float-mounted on adhesive-coated glass slides for Ki-67 and P53 staining. Primary antibodies included a monoclonal mouse antihuman P53 protein antibody (PAb1801; Novocastra, Newcastle, $\mathrm{UK})$ at 1:50 dilution for P53 and monoclonal mouse antihuman Ki-67 antibody (MIB-1; DAKO, Glostrup, Denmark) at 1:100 dilution for Ki-67. The DAKO EnVision system (DAKO EnVision labeled polymer, peroxidase) was used as the detection system for Ki-67. The streptavidin-biotin system (SAB-PO kit; Nichirei Co., Inc., Tokyo, Japan) was applied for the detection of the bound antibody of P53. Immunostained slides were scored after the entire slide had been evaluated by light microscopy. For all specimens, a negative control was adjacent to normal renal tissue specimen and the positive control was included in the staining kit (Figure 1-5).

\section{Immunostaining interpretation}

The level of Ki-67 and P53 expression in renal lupus lesions was evaluated according to the scoring system of Liang et al. ${ }^{9}$ Cell counts in immunohistochemically stained tissue sections were performed using a Carl Zeiss microscope $(\mathrm{GmbH}$, ena, Germany) with objective under $40 / 0.65 \times 10$ magnification. Cell nuclei were counted starting from the regions with the highest Ki-67 staining intensity. In all cases of LN, cells within the glomerular capillary tuft were counted without discriminating between endocapillary and extracapillary cells. Tubular cells were not included in the calculations. Labeled nuclei were counted by considering staining intensity. In each slide, three fields were selected, and in each field, approximately 100 cells were counted and the mean numbers of Ki-67- and P53-positive cells were recorded separately. The application of this system gives a score ranging from 0 to 3 for both degrees of positivity: percentage of positively stained cells $[(0,<1 \%),(1,1-10 \%),(2,10-50 \%),(3,>50 \%)]$ and degree

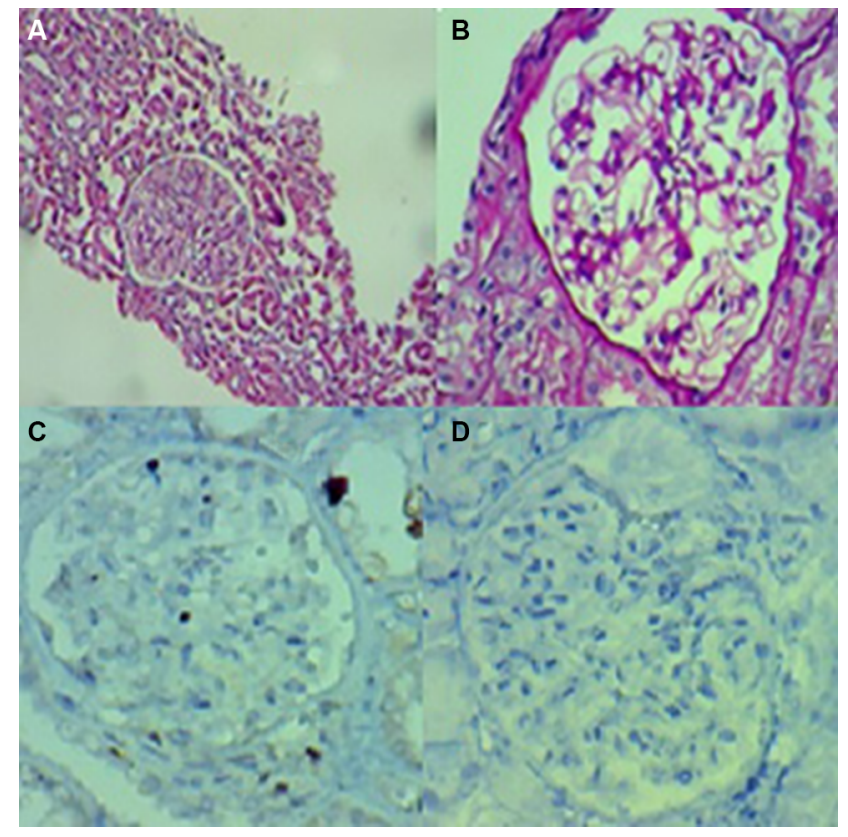

Figure I Light micrograph of a normocellular glomerulus and mesangial matrix and normal renal tubulointerstitium (negative control).

Notes: (A and B) Normal renal tissue. (H\&E stained, 200x). (C) P53 nuclear immunostaining, score 0 (200x). (D) Ki-67 nuclear immunostaining, score $0(200 \times)$.

of intensity of staining [( 0 , no staining), (1, faint staining), (2, chestnut brown), (3, deep brown)]. The sum score (positivity plus intensity) was then calculated for each specimen. Finally, the sum of the two scores was taken as a representative of the index of coexpression, which is a 0-6 expression score (low $<2$, moderate 2-4, high $>4$ ) (Figures 2-5).

\section{Statistical analysis}

Collected data were documented using the SPSS version 20.0 (IBM Corp., Armonk, NY, USA). Two types of statistics were created: 1) descriptive statistics: number, percentage, and mean \pm SD; 2) analytical statistics: Ki-67 and P53 indices of cLN with classes II, III, and IV LN were compared using the Mann-Whitney $U$-test, chi-squared test, Pearson chi-squared test, or Spearman Rho test. Level of significance was set at $P \leq 0.05$.

\section{Results}

Biopsy specimens of 33 children with LN have been enrolled. The distribution of renal biopsies according to ISN/RPS 2003 classification has been reported in Table 1. The mean total number of glomeruli per biopsy specimen was 13.6 \pm 10.2 (range: 5-38). The mean ESR was $22 \pm 16.5 \mathrm{~mm} / \mathrm{hr}$, which shows a positive correlation with subclasses of LN, which was higher in classes II, III, and IV $(P<0.01)$. The mean serum creatinine at the time of biopsy was $1.7 \pm 0.86 \mathrm{mg} / \mathrm{dL}$ (range: $0.6-6.2 \mathrm{mg} / \mathrm{dL}$ ) 


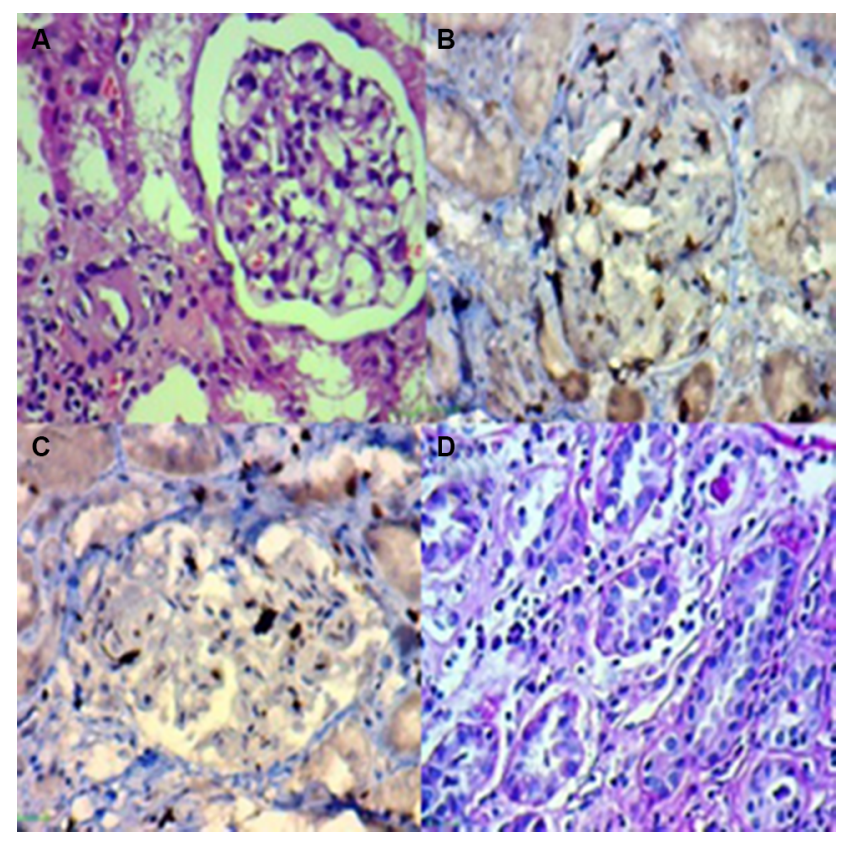

Figure 2 (A) Lupus nephritis class II. Light micrograph of a glomerulus with mild mesangial hypercellularity (H\&E stained, 200X). (B) P53 nuclear immunostaining of apoptotic cells in class II of cLN, score I (200X). (C) Ki-67 nuclear immunostaining of mesangial proliferative glomeruli in class II of cLN, score I (200x). (D) Mild mononuclear tubulointerstitial nephritis (H\&E stained, 200X).

Abbreviation: $\mathrm{cLN}$, childhood lupus nephritis.

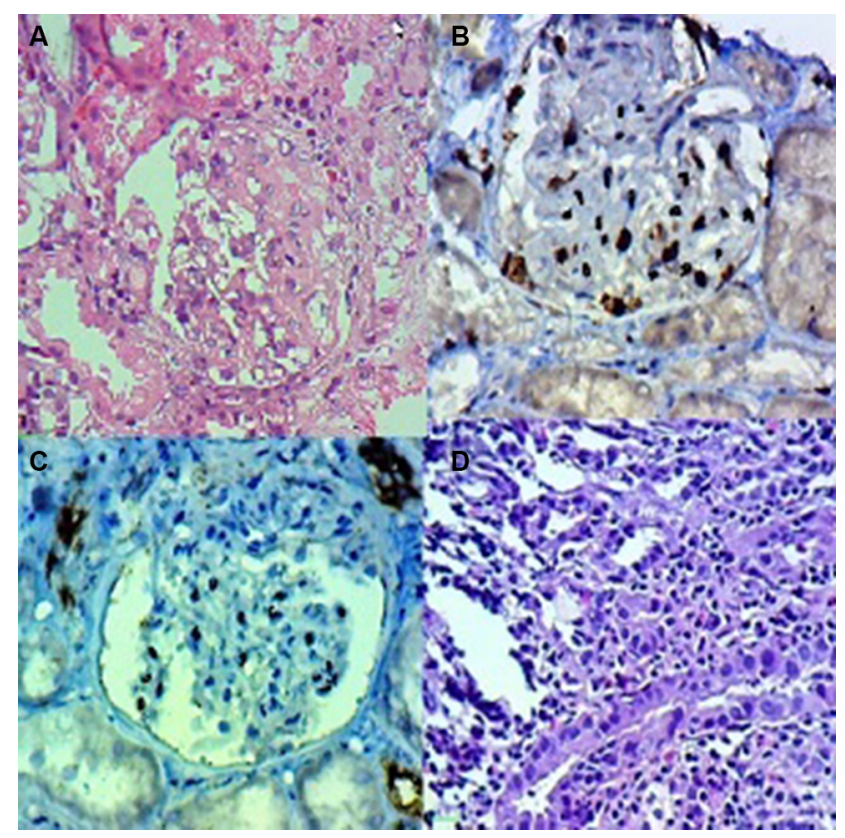

Figure 4 (A) A segment of glomerulus showing endocapillary hypercellularity, capillary wall double contours, and wire loop lesions (H\&E stained, 200X). (B) P53 nuclear immunostaining of apoptotic cells in class II of cLN, score II/III (200X). (C) $\mathrm{Ki}-67$ nuclear immunostaining of mesangial proliferative glomeruli in class II of cLN, score II/III (200x). (D) Moderate mononuclear tubulointerstitial nephritis (H\&E stained, 200x).

Abbreviation: $\mathrm{cLN}$, childhood lupus nephritis.

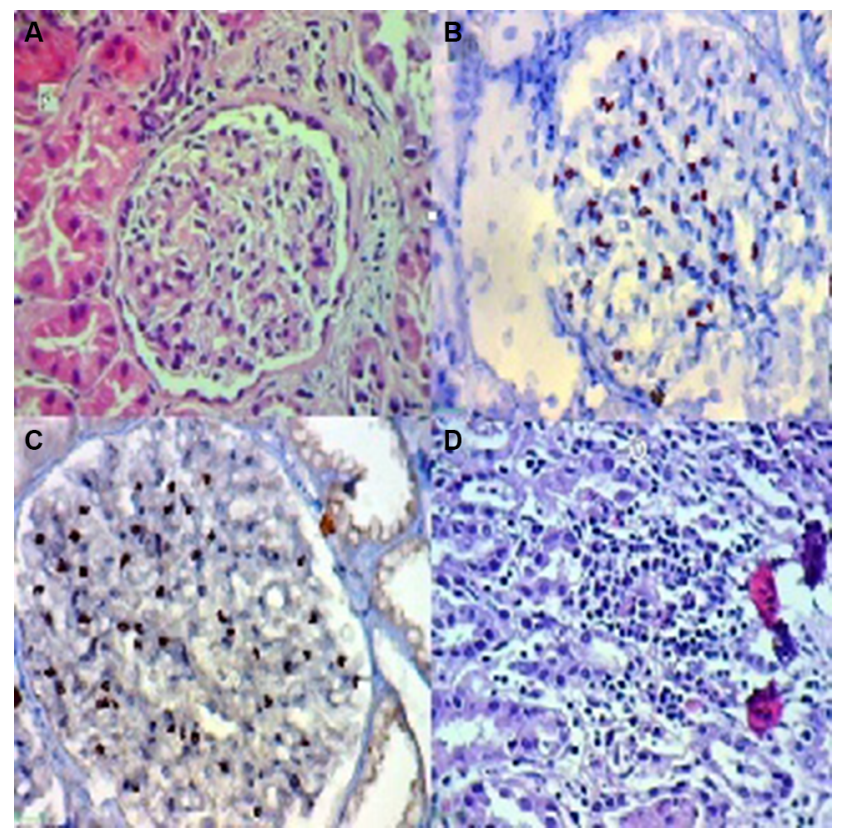

Figure 3 (A) Lupus nephritis class III. Light micrograph showing a glomerulus with segmental endocapillary hypercellularity, mesangial hypercellularity, and capillary wall thickening (H\&E stained, 200×). (B) P53 nuclear immunostaining of apoptotic cells in class II of cLN, score I/II (200X). (C) Ki-67 nuclear immunostaining of mesangial proliferative glomeruli in class II of cLN, score I/II (200×). (D) Mild to moderate mononuclear tubulointerstitial nephritis (H\&E stained, 200×).

Abbreviation: $\mathrm{cLN}$, childhood lupus nephritis.

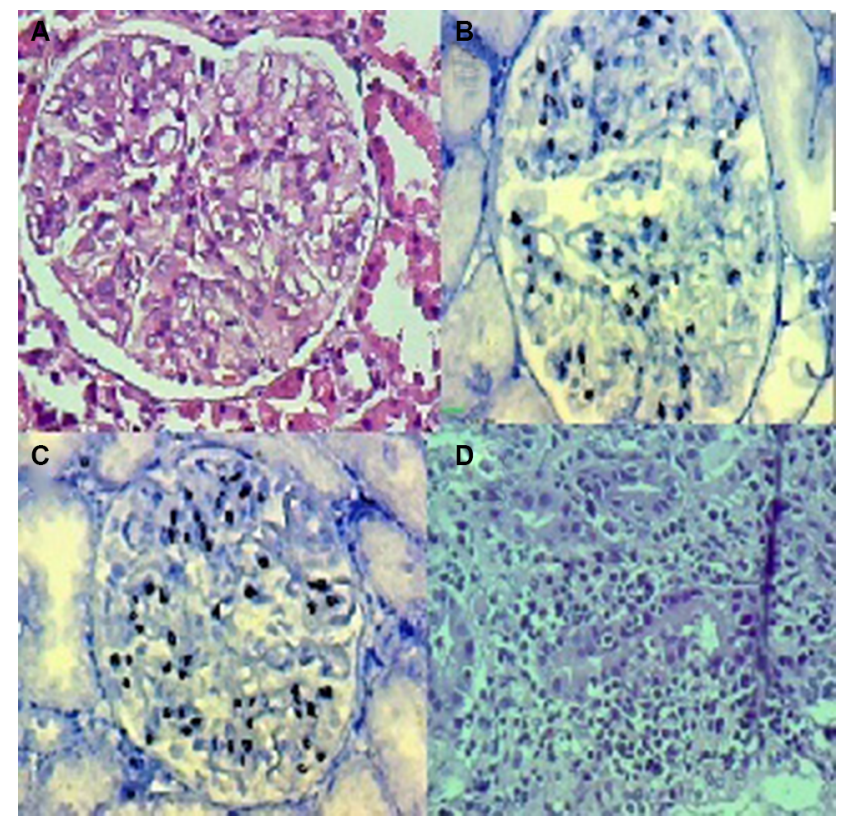

Figure 5 (A) Lupus nephritis class V. Light micrograph of a glomerulus with diffuse membranous glomerulonephritis (H\&E stained, 200x). (B) P53 nuclear immunostaining of apoptotic cells in class II of cLN, score II/III (200x). (C) Ki-67 nuclear immunostaining of mesangial proliferative glomeruli in class II of $\mathrm{cLN}$, score II/III (200x). (D) Moderate to severe mononuclear tubulointerstitial nephritis (H\&E stained, 200x).

Abbreviation: $\mathrm{cLN}$, childhood lupus nephritis. 
Table I Distribution of renal biopsies according to ISN/RPS 2003 classification

\begin{tabular}{|l|l|}
\hline ISN/RPS (scale) & Frequency \\
\hline Class II & $9(27.3 \%)$ \\
\hline Class III & $9(27.3 \%)$ \\
\hline Class IV & $9(27.3 \%)$ \\
\hline Class V & $6(18.1 \%)$ \\
\hline Total & $33(100 \%)$ \\
\hline
\end{tabular}

Abbreviation: ISN/RPS, International Society of Nephrology/Renal Pathology Society.

and the mean 24-hour urinary protein excretion was $2.8 \pm 1.1$ $\mathrm{g}$ (range: $250 \mathrm{mg}$ to $16.7 \mathrm{~g}$ ). The main demographic, clinical, and laboratory findings are presented in Table 2.

We tried to regard the activity and chronicity of glomerular lesions. Evaluation of biopsies using the NIH-AI revealed moderate activity (mean 7.94 \pm 6.3 , range $0-20$ ). Scoring of biopsies using the NIH chronicity score revealed substantial scarring (mean 4.85 \pm 4.37 , range $0-12$ ) at the time of biopsy. There were few discrepancies between clinical presentations and pathologic findings at renal biopsy in our patients with LN. For example, for $4 / 9$ patients in class II and $2 / 9$ patients in class III, serum creatinine was in the normal range. For $3 / 9$ patients in class II, urine protein was in the normal range. The mean serum creatinine level in class II was $0.8 \pm 0.32 \mathrm{mg} / \mathrm{dL}$, and in class III it was $1.2 \pm 0.7 \mathrm{mg} / \mathrm{dL}$ and was significantly higher in classes IV and $\mathrm{V}$, which was $1.8 \pm 0.7 \mathrm{mg} / \mathrm{dL}(P<0.05)$. The mean urine protein in class II was $0.6 \pm 0.46 \mathrm{~g} / 24$ hours and in class III it was $1.3 \pm 0.71 \mathrm{~g} / 24$ hours and was significantly higher in classes IV and V, which was $1.8 \pm 0.62 \mathrm{~g} / 24$ hours $(P<0.04)$. Also, the frequency of urine epithelial casts and hematuria in different $\mathrm{LN}$ classes has been mentioned in Table 3. The mean D-dimer level in class II was $1.07 \pm 0.43 \mu \mathrm{g} / \mathrm{mL}$ and in class III it was $3.2 \pm 1.59 \mu \mathrm{g} / \mathrm{mL}$ and was significantly higher in classes IV and V, which was $4.34 \pm 2.72 \mu \mathrm{g} / \mathrm{mL}$, and $4.39 \pm 3.10 \mu \mathrm{g} / \mathrm{mL}$, respectively $(P<0.05)$. A significant association was observed between the expression status of Ki-67 and that of P53 $(P<0.02)$. All results of correlation analysis between positive coexpression of Ki-67 and P53 with independent variables, such as demographic and laboratory findings, have been reported in Table 3. A significant positive correlation was revealed between the coexpression of Ki-67 and P53 indices with LN subclasses (II, III and IV), LN activity, ESR, D-dimer, serum creatinine, proteinuria, and anticardiolipin antibodies. Also, a significant adverse correlate ion was revealed between the coexpression of Ki-67 and P53 indices with serum complements. There was no correlation between sex, age, epithelial cast, and serologic markers with coexpression of Ki-67 and P53 indices (Table 3).

\section{Discussion}

Renal disease occurs in $50 \%-75 \%$ of all pediatric SLE patients, mostly within the first 2 years after diagnosis. LN is more common in male populations, ${ }^{6-8}$ which is verified in our study (male to female: 1.75:1). Initial manifestations of the renal disease are variable and could range from minimal proteinuria and microscopic hematuria to nephrotic range proteinuria, urinary casts, severe hypertension, peripheral edema, and renal insufficiency. Both glomeruli and renal interstitium are targeted in SLE. ${ }^{5}$ However, children with LN at onset, but who could not fulfill the SLE criterion, developed delayed symptoms over time. Therefore, data on the clinical course, long-term outcome, and predictors of disease progression in cLN are scant. The results of the Italian study ${ }^{10}$ indicate that LN has a worse prognosis in younger children and suggests that all patients with LN, regardless of the presence of other SLE symptoms, should be treated appropriately at the disease's onset to achieve complete remission before reaching the end-stage kidney disease. However, the role of the renal biopsy in the prediction of outcome and prognosis has not been clearly established. Several studies have focused on the discrepancies between clinical presentations and pathologic findings (based on ISN/RPS 2003 classification) in cLN. Many studies confirm that silent LN (lack of clinical presentation) has been reported in classes II and IV LN. ${ }^{1-19}$ Patients with low-level proteinuria (<1 g/24 hours) and without microscopic hematuria were reported in classes III or IV..$^{20,21}$ Nephrotic range proteinuria has been reported in class V and even in classes II and III LN. ${ }^{22,23}$ Our patients showed a few discrepancies between clinical presentation and pathologic findings. We found $1 / 9$ patients with microscopic hematuria; $3 / 9$ children with proteinuria; 2/9 patients with epithelial casts; and 2/9 patients with high serum creatinine in class II patients, but Ki-67 and P53 expression showed a low range in them. Interestingly, in class III, all patients showed high serum creatinine, but the $\mathrm{Ki}-67$ index was moderate in $8 / 9$ patients. It is true that early clinical and histologic diagnosis of $\mathrm{LN}$ is pivotal and critical in order to minimize the risk of progression to end stage renal disease. ${ }^{5}$ Recent studies among the adult population directly show that the $\mathrm{Ki}-67$ proliferation index is correlated with the type of LN, disease activity, and some laboratory findings in adult population directly. ${ }^{24,25}$ In the Iranian adult population, a significant, positive correlation between serum creatinine with percent activity of LN (and also between proteinuria with percent chronicity of LN) has been reported. ${ }^{25}$ We found the same results in cLN. Those results reveal that there is a significant positive association between the Ki-67 proliferation index with ESR, serum 


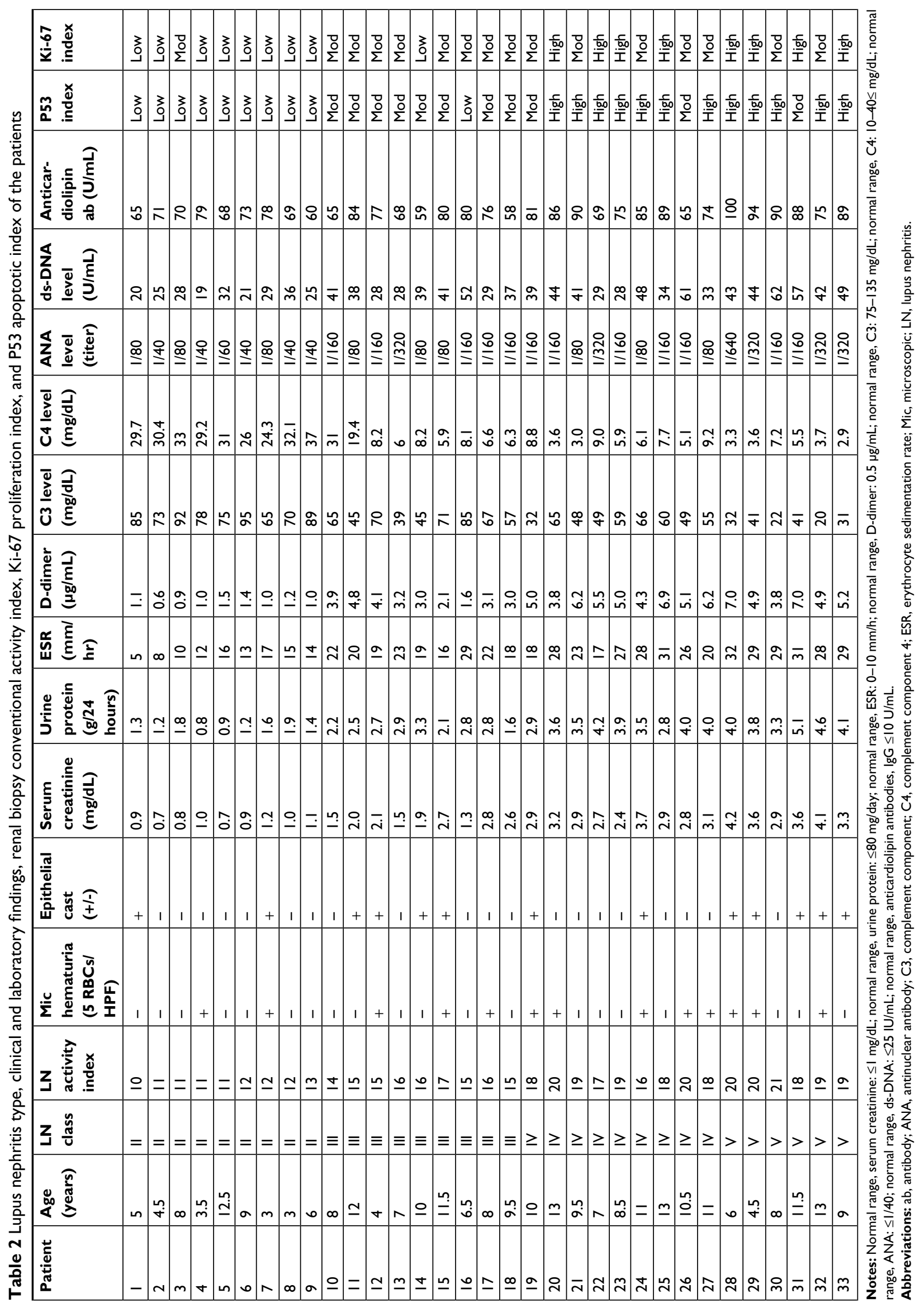


Table 3 Correlation between P53 and Ki-67 coexpression and separately with demographic and laboratory findings

\begin{tabular}{|c|c|c|c|c|}
\hline Parameters & Ki-67 (P-value) & P53 (P-value) & Ki-67 and P53 (P-value) & $\mathbf{R R}$ \\
\hline Age (years) & $<0.41$ & $<0.10$ & $<0.31$ & 0.54 \\
\hline Gender & $<0.08$ & $<0.06$ & $<0.09$ & 0.74 \\
\hline LN subclasses (II, III, IV, V) & $<0.02$ & $<0.04$ & $<0.02$ & 0.64 \\
\hline Glomerular activity index & $<0.04$ & $<0.03$ & $<0.03$ & 0.55 \\
\hline ESR & $<0.04$ & $<0.05$ & $<0.04$ & 0.86 \\
\hline D-dimer $(\mu \mathrm{g} / \mathrm{mL})$ & $<0.03$ & $<0.03$ & $<0.03$ & 0.52 \\
\hline Serum creatinine $(\mathrm{mg} / \mathrm{dL})$ & $<0.01$ & $<0.04$ & $<0.03$ & 0.48 \\
\hline Urine protein (g/24 hours) & $<0.03$ & $<0.04$ & $<0.04$ & 0.66 \\
\hline C3 level (mg/dL) & $<0.05$ & $<0.04$ & $<0.02$ & 0.81 \\
\hline C4 level (mg/dL) & $<0.03$ & $<0.03$ & $<0.03$ & 0.74 \\
\hline ANA (titer) & $<0.14$ & $<0.21$ & $<0.18$ & 0.68 \\
\hline Ds-DNA (U/mL) & $<0.95$ & $<0.23$ & $<0.53$ & 0.58 \\
\hline Anticardiolipin lgG (U/mL) & $<0.05$ & $<0.05$ & $<0.05$ & 0.62 \\
\hline Mic hematuria (5 RBC/HPF) & $<0.14$ & $<0.66$ & $<0.38$ & 0.73 \\
\hline Epithelial cast $(+/-)$ & $<0.67$ & $<0.92$ & $<0.8$ I & 0.68 \\
\hline
\end{tabular}

Abbreviation: ANA, antinuclear antibody C3, complement component 3; C4, complement component 4; ESR, erythrocyte sedimentation rate; LN, lupus nephritis.

creatinine, the degree of proteinuria, activity index, and negative association with complements. No correlation was noted between the Ki-67 proliferation index and hematuria. One study in Iran was conducted on 29 adults. Researchers did not find a correlation between anticardiolipin antibody positivity with the Ki-67 proliferation index. Also, no correlation was found between serum complements $\mathrm{C} 3$ and $\mathrm{C} 4$ levels with the Ki-67 proliferation index. Also, both LN activity index and Ki-67 proliferation index show a significantly rising slope in high classes of 29 adult patients who were diagnosed for class III and class IV LN. ${ }^{25}$ There is only one study that worked on immunohistochemical expression of Ki-67 and P53 in cutaneous lupus erythematosus. They reported that P53-induced apoptosis accompanied with Ki-67-induced hyperproliferation and may play a part in the pathogenesis of discoid lupus erythematosus. ${ }^{26}$ In our study, the LN activity index was low $(\leq 3)$ in patients with class II LP and also Ki-67 and P53 coexpression was low, which was indicated in the role of proliferation and apoptotic indices as a predictive factor in cLN. Also, all patients with class IV showed a high Ki-67 and P53 coexpression. Therefore, the rising slope of $\mathrm{Ki}-67$ and P53 coexpression parallel to developing classes of LN and the activity index were highlighted as possible pathogenesis effects of expression of these markers.

\section{Conclusions}

The current study demonstrated that cellular proliferation and apoptosis may play a part in the pathogenesis of cLN through the tissue expression of Ki-67 and P53. The coexpression of Ki-67 and P53 is correlated with disease classes II, III, and IV $\mathrm{LN}$, renal biopsy activity index, and also some functional and clinical variables. Therefore, future studies will focus on the standardization of Ki-67 and P53 assessment in renal activity index parameters and histopathology subclasses of cLN.

\section{Acknowledgments}

The authors thank the personnel of Iran University of Medical Sciences Hospital and Ali Asghar Children Hospital in Tehran for participation in the completion checklists.

\section{Disclosure}

The authors report no conflicts of interest in this work.

\section{References}

1. Perfumo F, Martini A. Lupus nephritis in children. Lupus. 2005;14(1): 83-88.

2. Levy DM, Kamphuis S. Systemic lupus erythematosus in children and adolescents. Pediatr Clin North Am. 2012;59(2):345-364.

3. Bernatsky S, Boivin JF, Joseph L, et al. Mortality in systemic lupus erythematosus. Arthritis Rheum. 2006;54(8):2550-2557.

4. Alarcón GS, Mcgwin G, Petri M, et al. Baseline characteristics of a multiethnic lupus cohort: PROFILE. Lupus. 2002;11(2):95-101.

5. Austin HA, Boumpas DT, Vaughan EM, Balow JE. Predicting renal outcomes in severe lupus nephritis: contributions of clinical and histologic data. Kidney Int. 1994;45(2):544-550.

6. Bihl GR, Petri M, Fine DM. Kidney biopsy in lupus nephritis: look before you leap. Nephrol Dial Transplant. 2006;21(7):1749-1752.

7. Sada KE, Makino H. Usefulness of ISN/RPS classification of lupus nephritis. J Korean Med Sci. 2009;24(Supp1):S7-S10.

8. Leehey DJ, Katz AI, Azaran AH, Aronson AJ, Spargo BH. Silent diffuse lupus nephritis: long-term follow-up. Am JKidney Dis. 1982;2(1 Suppl 1): $188-196$.

9. Liang MH, Socher SA, Larson MG, Schur PH. Reliability and validity of six systems for the clinical assessment of disease activity in systemic lupus erythematosus. Arthritis Rheum. 1989;32(9):1107-1118.

10. Ruggiero B, Vivarelli M, Gianviti A, et al. Lupus nephritis in children and adolescents: results of the Italian Collaborative Study. Nephrol Dial Transplant. 2013;28(6):1487-1496. 
11. Bernatsky S, Boivin JF, Joseph L, et al. Mortality in systemic lupus erythematosus. Arthritis Rheum. 2006;54(8):2550-2557.

12. Faurschou M, Starklint H, Halberg P, Jacobsen S. Prognostic factors in lupus nephritis: diagnostic and therapeutic delay increases the risk of terminal renal failure. J Rheumatol. 2006;33(8):1563-1569.

13. Nossent JC, Henzen-Logmans SC, Vroom TM, Huysen V, Berden JH, Swaak AJ. Relation between serological data at the time of biopsy and renal histology in lupus nephritis. Rheumatol Int. 1991;11(2):77-82.

14. Gladman DD, Urowitz MB, Cole E, Ritchie S, Chang CH, Churg J. Kidney biopsy in SLE. I. A clinical-morphologic evaluation. $Q J \mathrm{Med}$. 1989;73(272):1125-1133.

15. Font J, Torras A, Cervera R, Darnell A, Revert L, Ingelmo M. Silent renal disease in systemic lupus erythematosus. Clin Nephrol. 1987;27(6):283-288.

16. Gonzalez-Crespo MR, Lopez-Fernandez JI, Usera G, Poveda MJ, Gomez-Reino JJ. Outcome of silent lupus nephritis. Semin Arthritis Rheum. 1996;26(1):468-476.

17. Zabaleta-Lanz M, Vargas-Arenas RE, Tápanes F, Daboin I, Atahualpa Pinto J, Bianco NE. Silent nephritis in systemic lupus erythematosus. Lupus. 2003;12:26-30.

18. Moroni G, Pasquali S, Quaglini S, et al. Clinical and prognostic value of serial renal biopsies in lupus nephritis. Am J Kidney Dis. 1999;34(3):530-539.
19. Bennett WM, Bardana EJ, Norman DJ, Houghton DC. Natural history of "silent" lupus nephritis. Am J Kidney Dis. 1982;1(6):359-363.

20. Christopher-Stine L, Siedner M, Lin J, et al. Renal biopsy in lupus patients with low levels of proteinuria. J Rheumatol. 2007;34(2):332-335.

21. Hsieh YP, Wen YK, Chen ML. The value of early renal biopsy in systemic lupus erythematosus patients presenting with renal involvement. Clin Nephrol. 2012;77(1):18-24.

22. Stankeviciute N, Jao W, Bakir A, Lash JP. Mesangial lupus nephritis with associated nephrotic syndrome. J Am Soc Nephrol. 1997;8(7): 1199-1204

23. Han TS, Schwartz MM, Lewis EJ. Association of glomerular podocytopathy and nephrotic proteinuria in mesangial lupus nephritis. Lupus. 2006;15(2):71-75.

24. Cheah PL, Kunaseegaran R, Looi LM. Expression of proliferating cell associated protein, $\mathrm{Ki}-67$, supports cellular proliferation in WHO Class IV lupus nephritis. Malays J Pathol. 2001;23(1):27-30.

25. Dalkilic E, Filiz G, Yavuz M, et al. Ki-67 proliferation index in renal biopsy samples of patients with systemic lupus erythematosus and its correlation with clinical findings. Iran J Kidney Dis. 2013;7(3):198-203.

26. Seleit IA, Asaad N, Maree A, Wahed MA. Immunohistochemical expression of $\mathrm{p} 53$ and $\mathrm{Ki}-67$ in cutaneous lupus erythematosus. J Egypt Women Dermatol Soc. 2010;7:5-15.

\section{Publish your work in this journal}

The International Journal of Nephrology and Renovascular Disease is an international, peer-reviewed open access journal focusing on the pathophysiology of the kidney and vascular supply. Epidemiology, screening, diagnosis, and treatment interventions are covered as well as basic science, biochemical and immunological studies. The manuscript management system is completely online and includes a very quick and fair peer-review system, which is all easy to use. Visit http://www. dovepress.com/testimonials.php to read real quotes from published authors. 